Supporting Information for

\title{
Fluorophilic Ionophores for Potentiometric pH Determinations with Fluorous Membranes of Exceptional Selectivity
}

Paul G. Boswell, ${ }^{\dagger}$ Csongor Szíjjártóo, ${ }^{\S}$ Markus Jurisch, ${ }^{\ddagger}$ John A Gladysz, ${ }^{\ddagger \dagger}$ József Rábai,${ }^{\S}$ and Philippe Bühlmann ${ }^{*} \dagger$

University of Minnesota, Department of Chemistry 207 Pleasant Street SE, Minneapolis, Minnesota 55455, Institut für Organische Chemie and Interdisciplinary Center for Molecular Materials, FriedrichAlexander Universität, Erlangen-Nürnberg, Henkestrasse 42, 91054 Erlangen, Germany, Department of Chemistry, Texas A\&M University, PO Box 30012, College Station, Texas 77842-3012, and Institute of Chemistry, Eötvös Loránd University, P.O. Box 32, H-1518 Budapest 112, Hungary

buhlmann@chem.umn.edu

${ }^{\dagger}$ University of Minnesota

${ }^{\ddagger}$ Friedrich-Alexander Universität

${ }^{\S}$ Eötvös Loránd University

${ }^{f}$ Texas A\&M University

Details of Potentiometric Measurements 
In the case of $\mathrm{Na}^{+}$and $\mathrm{K}^{+}$selectivity measurements, three solutions of different $\mathrm{pH}$ but equal cation concentrations were prepared prior to measurements: the first contained $1.0 \mathrm{M}$ of the hydroxide salt of the primary ion, the second solution contained $0.316 \mathrm{M}$ of the hydroxide salt of the primary ion and $0.684 \mathrm{M}$ of the chloride salt of the primary ion, and the third solution contained $0.1 \mathrm{M}$ of the hydroxide salt of the primary ion, $0.9 \mathrm{M}$ of the chloride salt of the primary ion, and $10 \mathrm{mM}$ tris(hydroxymethyl)aminomethane (TRIS) buffer. In a fixed interference experiment, the electrode response was recorded as the electrodes were placed in the first, the second, and then the third solution. At that point, a solution containing $1.0 \mathrm{M} \mathrm{HCl}$ and $1.0 \mathrm{M}$ of either $\mathrm{KCl}$ or $\mathrm{NaCl}$ was added incrementally to decrease in situ the $\mathrm{pH}$ of that third solution. In the case of $\mathrm{Ca}^{2+}$ selectivity measurements, the starting solution consisted of $1.0 \mathrm{M} \mathrm{CaCl}_{2}$ and $10 \mathrm{mM}$ TRIS buffer. To that, a $1.0 \mathrm{M}$ $\mathrm{LiOH}$ solution was added incrementally to increase the $\mathrm{pH}$ until a precipitate was observed. In another experiment, to decrease the $\mathrm{pH}$, a solution of $1.0 \mathrm{M} \mathrm{CaCl}_{2}$ and $1.0 \mathrm{M} \mathrm{HCl}$ was incrementally added to a starting solution of $1.0 \mathrm{M} \mathrm{CaCl}_{2}$ and $10 \mathrm{mM} \mathrm{Ca}(\mathrm{OH})_{2}$. An InLab $201 \mathrm{pH}$ half-cell glass electrode (Mettler Toledo, Columbus, $\mathrm{OH}$ ) was used to determine the $\mathrm{H}^{+}$activity for all selectivity measurements and calibration curves. The glass electrode was calibrated by a seven-point calibration with buffer reference solutions of $\mathrm{pH} 4.00,7.00,10.01,12.00,13.00,13.1$, and 13.7. The $\mathrm{pH} 13.7$ and 13.1 buffers were prepared as described previously ${ }^{1}$ by making a $0.8 \mathrm{M}$ tetramethylammonium hydroxide, $0.1 \mathrm{M}$ tetramethylammonium chloride solution, and a $0.19 \mathrm{M}$ tetramethylammonium hydroxide, $0.1 \mathrm{M}$ tetramethylammonium chloride solution, respectively; their approximate $\mathrm{pH}$ values were calculated with the extended Debye-Hückel formalism. All other activities were calculated according to a twoparameter Debye-Hückel approximation. $^{2}$

\section{Ion-Pair Association Constant Calculations}

The relationship between the molar conductivity, $\Lambda$, and the salt concentration in $\mathbf{2}, c$, can be modeled with the Fuoss-Kraus theory ${ }^{3}$ 


$$
\Lambda=\frac{\Lambda_{\mathrm{s}}^{\infty}}{c^{1 / 2} K_{\mathrm{ip}}^{1 / 2}}+\frac{\Lambda_{\mathrm{t}}^{\infty} c^{1 / 2} K_{\mathrm{t}}}{K_{\mathrm{ip}}^{1 / 2}}
$$

where $\Lambda_{\mathrm{s}}^{\infty}$ and $\Lambda_{\mathrm{t}}^{\infty}$ are the free electrolyte and triple ion electrolyte limiting molar conductivities, and $K_{\text {ip }}$ and $K_{\mathrm{t}}$ are the ion pair and triple ion formation constants. The common approximation of $\Lambda_{\mathrm{t}}^{\infty}$ with $2 \Lambda_{\mathrm{s}}^{\infty} / 3$ yields: ${ }^{4}$

$$
\Lambda=\frac{\Lambda_{\mathrm{s}}^{\infty}}{c^{1 / 2} K_{\mathrm{ip}}^{1 / 2}}+\frac{2 \Lambda_{\mathrm{s}}^{\infty} c^{1 / 2} K_{\mathrm{t}}}{3 K_{\mathrm{ip}}^{1 / 2}}
$$

More complex modeling of the system is available that can take into account formation of quadrupoles, viscosity changes, and ion activities, but since a decrease in the slope at higher concentrations was not observed for any of the salts, these effects seemed insignificant here.

Because the limiting molar conductivities of the salts used in this work have not been measured in any other solvents, Walden's rule could not be used to determine the limiting molar conductivities in perfluoroperhydrophenanthrene. Instead, the Stokes-Einstein law was used to approximate the limiting molar conductivities of the individual ions

$$
\lambda_{i}^{\infty}=\frac{z_{i}^{2} F^{2}}{6 N_{\mathrm{A}} \pi \eta r_{i}}
$$

where $F$ is Faraday's constant, $N_{\mathrm{A}}$ is Avogadro's number, $r_{i}$ is the Stokes radius of the ion, $\eta$ is the viscosity of the solvent $\left(25^{\circ} \mathrm{C}, 28.4 \mathrm{cP}\right),{ }^{5}$ and $\lambda_{i}^{\infty}$ is the limiting molar conductivity of the free ion. The sum of the limiting molar conductivities of the free cation, $\lambda_{+}^{\infty}$, and anion, $\lambda_{-}^{\infty}$, gives the limiting molar conductivity of the electrolyte.

$$
\Lambda_{\mathrm{s}}^{\infty}=\lambda_{+}^{\infty}+\lambda_{-}^{\infty}
$$

The Stokes radii used for these measurements were either approximated by crystallographic radii, ${ }^{6}$ or calculated from the work of Edward. ${ }^{7}$ They are listed in Table 1 along with the calculated ion-pair and triple ion formation constants of each salt of tetrakis[3,5-bis(perfluorohexyl)phenyl]borate. 
Table 1. Ion-pair and triple ion formation constants measured for the tetrakis[3,5bis(perfluorohexyl)phenyl]borate salts of various cations, along with the ionic radius used for each calculation.

\begin{tabular}{llll}
\hline Cation & $\log \left[K_{\mathrm{ip}}\right] / \mathrm{M}^{-1}$ & $\begin{array}{l}\log \left[K_{\mathrm{t}}\right] \\
\mathrm{M}^{-1}\end{array}$ & $r_{+}(\AA)^{\mathrm{a}}$ \\
\hline $\mathrm{H}_{3} \mathrm{O}^{+}$ & 14.77 & $<2$ & $1.30^{\mathrm{b}}$ \\
$\mathrm{Li}^{+}$ & 14.61 & 2.88 & $0.69^{\mathrm{b}}$ \\
$\mathrm{Na}^{+}$ & 14.28 & 3.15 & $1.02^{\mathrm{b}}$ \\
$\mathrm{K}^{+}$ & 14.49 & 3.77 & $1.38^{\mathrm{b}}$ \\
$\mathrm{Cs}^{+}$ & 14.28 & 2.02 & $1.70^{\mathrm{b}}$ \\
$\mathrm{NH}_{4}^{+}$ & 14.13 & 2.53 & $1.48^{\mathrm{b}}$ \\
$\mathrm{PPh}_{4}^{+}$ & 14.20 & 4.93 & $4.24^{\mathrm{b}}$ \\
$\mathrm{Ionophore} \mathrm{3-H}^{+}$ & 15.79 & 4.48 & $5.61^{\mathrm{c}}$ \\
Ionophore 4- $\mathrm{H}^{+}$ & 16.24 & 4.84 & $5.20^{\mathrm{c}}$ \\
Ionophore 5- $-\mathrm{H}^{+}$ & 15.15 & 4.25 & $5.76^{\mathrm{c}}$ \\
Ionophore 6- $\mathrm{H}^{+}$ & 14.05 & 3.91 & $5.99^{\mathrm{c}}$
\end{tabular}

\footnotetext{
${ }^{a}$ The value of $r_{-}$calculated for tetrakis[3,5-bis(perfluorohexyl)phenyl]borate was $7.396 \AA$.

${ }^{\mathrm{b}}$ Ionic crystal radius. ${ }^{6}$

${ }^{\mathrm{c}}$ Calculated ionic radius. ${ }^{7}$
}

The ion-pair and triple ion formation constants reported in this paper are smaller than our previously reported values. ${ }^{8}$ The new values are closer to those of similar salts in cyclohexane. For example, tetradecylammonium tetrakis(4-fluorophenyl)borate in cyclohexane was reported to have a $\log \left(K_{\text {ip }}\right)$ of 10.43 and a $\log \left(K_{\mathrm{t}}\right)$ of $2.72 .{ }^{9}$ Tridodecylammonium tetraphenylborate in cyclohexane was reported to have a $\log \left(K_{\mathrm{ip}}\right)$ of 13.21 and a $\log \left(K_{\mathrm{t}}\right)$ of $3.44 .{ }^{9}$ On one hand, we recorded more data points for some 
of the values presented here, increasing their accuracy. More importantly, we corrected a calculation error that had affected the previously reported values. ${ }^{8}$

\section{Derivation of Equation 1}

The selectivity coefficient for an ion-exchanger membrane $K_{J I}^{p o t}(I E)$ containing no ionophore is given by $^{10}$

$$
K_{J I}^{p o t}(I E)=\frac{\left[J^{+}(J)\right]^{z}}{K_{J I}\left[I^{z+}(I)\right]}
$$

where $I^{z+}$ is the ion of charge $\mathrm{z}$ that the ionophore can bind (in our case, $\mathrm{H}^{+}$), $J^{+}$is the reference ion that we assume does not interact with the ionophore $\left(\mathrm{PPh}_{4}^{+}\right),\left[I^{z+}(I)\right]$ is the concentration of free $I^{z+}$ in the membrane phase while the membrane is responding only to $I^{z+},\left[J^{+}(J)\right]$ is the concentration of free $J^{+}$in the membrane while the membrane is responding only to $J^{+}$, and $K_{I J}$ is the equilibrium constant for the ion exchange of $I^{z+}$ and $J^{+}$between the membrane and sample phases. Since there is no ionophore in the membrane, $\left[J^{+}(J)\right]$ is equal to $R_{\mathrm{T}}$, the total concentration of ionic site in the membrane, and $\left[I^{z+}(I)\right]$ is equal to $R_{\mathrm{T}} / z$.

$$
K_{J I}^{p o t}(I E)=\frac{z R_{\mathrm{T}}{ }^{z}}{K_{J I} R_{\mathrm{T}}}
$$

If ion-pair association constants were considered in this theory, the assumptions that $\left[J^{+}(J)\right]=R_{\mathrm{T}}$ and $\left[I^{z+}(I)\right]=R_{\mathrm{T}} / z$ would not be true. Ion-pair association is quite relevant to our system, but it was already accounted for by correcting the selectivity coefficients.

Similarly, the selectivity coefficient of the equivalent ionophore-doped membrane is given by

$$
K_{J I}^{p o t}(L)=\frac{R_{\mathrm{T}}{ }^{z}}{K_{J I}\left[I^{z+}(I)\right]}
$$

where only $\left[J^{+}(J)\right]$ is equal to $R_{\mathrm{T}}$ since it is assumed that $J^{+}$does not interact with the ionophore. Combining equations S6 and S7 gives 


$$
\left[I^{z+}(I)\right]=\frac{R_{\mathrm{T}}}{z} \frac{K_{J I}^{p o t}(I E)}{K_{J I}^{p o t}(L)}
$$

If a 1:1 stoichiometry is assumed, the binding constant of the ionophore with $I^{z+}, \beta_{I L}$, is given by

$$
\beta_{I L}=\frac{\left[L I^{z+}\right]}{[L]\left[I^{z+}\right]}
$$

where $[L]$ is the concentration of unbound ionophore and $\left[L I^{z+}\right]$ is the concentration of the ionophore$I^{z+}$ complex in the membrane. Substituting equation S9 into equation S8 and solving for $\beta_{I L}$ gives

$$
\beta_{I L}=\left(\frac{K_{J I}^{p o t}(L)}{K_{J I}^{p o t}(I E)}\right) \frac{z\left[L I^{z+}\right]}{R_{\mathrm{T}}[L]}
$$

Since we cannot make the assumption that $\left[I^{z+}\right]$ is insignificant compared to $R_{\mathrm{T}}$ or the total concentration of ionophore (bound and unbound), $L_{\mathrm{T}}$

$$
\left[I L^{+}\right]=R_{\mathrm{T}}-\left[I^{z+}\right]
$$

and

$$
[L]=L_{\mathrm{T}}-R_{\mathrm{T}}+\left[I^{z+}\right]
$$

By combining equations $\mathrm{S} 8, \mathrm{~S} 10, \mathrm{~S} 11$, and $\mathrm{S} 12$, the final equation is derived.

$$
\beta_{I L}=\left(\frac{K_{J I}^{p o t}(L)}{K_{J I}^{p o t}(I E)}\right) \frac{z K_{J I}^{p o t}(L)-K_{J I}^{p o t}(I E)}{L_{\mathrm{T}} K_{J I}^{p o t}(L)-R_{\mathrm{T}} K_{J I}^{p o t}(L)+\frac{R_{\mathrm{T}}}{z} K_{J I}^{p o t}(I E)}
$$

Equation S13 applied to $\mathrm{H}^{+}$corresponds to equation 1 in the manuscript. Similar equations ${ }^{11-13}$ have been derived by Pretsch and Bakker to calculate ionophore binding constants, but in each case, the assumption is made that the ionophore binding is very strong, such that $\left[I L^{+}\right] \approx R_{\mathrm{T}}$, and $[L] \approx L_{\mathrm{T}}-R_{\mathrm{T}}$. Here, an equation was derived that is applicable even when ionophore binding is weak. 


\section{References}

1. Covington, A. K.; Bütikofer, H. P.; Camoes, M. F. G. F. C.; Ferra, M. I. A.; Rebelo, M. J. F. Pure Appl. Chem. 1985, 57, 887-898.

2. Meier, P. C. Anal. Chim. Acta 1982, 136, 363-368.

3. Kraus, C. A.; Fuoss, R. M. J. Am. Chem. Soc. 1933, 55, 21-36.

4. Izutsu, K. Electrochemistry in Nonaqueous Solutions; Wiley: Weinheim, 2002.

5. Maciejewski, A. J. Photochem. Photobiol. A 1990, 51.

6. $\quad$ Marcus, Y. Ion Properties; Marcel Dekker: New York, 1997.

7. Edward, J. T. J. Chem. Educ. 1970, 47, 261-270.

8. Boswell, P. G.; Bühlmann, P. J. Am. Chem. Soc. 2005, 127, 8958-8959.

9. Abbott, A. P.; Griffith, G. A.; Harper, J. C. Journal of the Chemical Society, Faraday Transactions 1997, 93, 577-582.

10. Bühlmann, P.; Pretsch, E.; Bakker, E. Chem. Rev. 1998, 98, 1593-1687.

11. Bakker, E.; Pretsch, E. J. Electrochem. Soc. 1997, 144, L125-L127.

12. Bakker, E.; Pretsch, E. Anal. Chem. 1998, 70, 295-302.

13. Ceresa, A.; Pretsch, E. Anal. Chim. Acta 1999, 395, 41-52. 